\title{
Inteligência Emocional e Provas de Raciocínio: Um Estudo Correlacional
}

\section{Emotional Intelligence and Reasoning Tests: A Correlacional Study}

\author{
Adauto Garcia de Jesus Junior ${ }^{*} a$ \& Ana Paula Porto Noronha ${ }^{b}$ \\ ${ }^{a}$ Centro Universitário Adventista de São Paulo, São Paul, Brasil \\ ${ }^{b}$ Universidade São Francisco, Itatiba, Brasil
}

\begin{abstract}
Resumo
O presente estudo propôs-se a investigar a validade convergente do Mayer, Salovey e Caruso Emotional Intelligence Test - MSCEIT, um instrumento de avaliação da Inteligência Emocional, por meio da correlação com as Baterias de Provas de Raciocínio - BPR-5, de inteligência fluida e cristalizada. A amostra consistiu de 191 universitários, com idades variando entre 17 e 60 anos, provenientes dos cursos de Pedagogia, Educação Física, Administração, Ciências contábeis e Sistemas de Informação de uma instituição particular do interior do estado de São Paulo. Os instrumentos utilizados foram aplicados em duas sessões coletivas em grupos separados por seus cursos. A correlação geral entre MSCEIT e BPR-5 foi baixa e significativa $(r=0,18 ; \mathrm{p}<0,05)$ indicando certa convergência de construtos. No entanto, essa correlação aumentou $(r=0,28 ; \mathrm{p}<0,01)$ quando suprimidas as provas de Raciocínio mecânico e espacial (BPR-5), pelo fato de avaliarem outras habilidades além da inteligência fluida e cristalizada.

Palavras-chave: Testes psicológicos; avaliação psicológica; validade convergente; inteligência.

Abstract

This paper intended to study the convergent validity as detailed by Mayer, Salovey and Caruso Emotional Intelligence Test - MSCEIT, as a tool to assess Emotional Intelligence by relating them to the Baterias de Provas de Raciocínio (Series of Reasoning Tests) - BPR-5, regarding fluid and crystallized intelligence. The sample consisted of $191 \mathrm{grad}$ students, between 17 and 60 years old, with majors in the areas of Pedagogy, PE, BA, Accounting and Information Systems, from a privately funded university from the state of Sao Paulo. The instruments were applied in two collective sessions in groups classified by their majors. The general correlation between MSCEIT and BPR- 5 was low and significant $(r=0,18 ; \mathrm{p}<0,05)$, showing a little convergence of constructs. However, this correlation was increased $(r=0,28 ; \mathrm{p}<0,01)$, when discarding the Spatial and Mechanical reasoning tests (BPR-5), for they also assess other skills besides Crystallized and Fluid Intelligence.

Keywords: Psychological tests; psychological assessment; convergent validity; intelligence.
\end{abstract}

A avaliação psicológica teve sua origem no contexto do diagnóstico e da rotulação de doentes mentais, o que lhe conferiu um início conturbado pelas críticas relacionadas à sua falta de critério e rigor científico (Anastasi \& Urbina, 2000). Essa tendência permaneceu ao longo de várias décadas nos meios profissional e científico. Atualmente, no entanto, ela conta com alta consideração e importância na atuação do psicólogo, tanto em termos nacionais quanto internacionais (Noronha \& Alchieri, 2002).

Há no Brasil problemas relacionados à construção e utilização de testes psicológicos, como analisados por Noronha (2002), que podem ser considerados decorrentes da ausência de pesquisa sobre a qualidade dos instrumentos comercializados. Para resgatar a confiabilidade dos testes frente a este quadro de despreparo e críticas, têm sido empregados importantes esforços de âmbito nacio-

* Endereço para correspondência: Rua Galileu Galilei, 854, Apto. 12, Jd. Irajá, Ribeirão Preto, SP, 14020-620. Tel: (16) 3021-5684. E-mail: adauto.garcia@unasp.edu.br nal e internacional, tanto por parte de pesquisadores, como de órgãos de regulamentação e fiscalização da atuação psicológica. Na Europa, diretrizes de uso de instrumentos psicológicos foram publicadas pela International Test Comission (ITC, 2001), a fim de orientar os profissionais quanto à seleção e administração dos instrumentos disponíveis. Nessa mesma direção, nos EUA, a American Educational Research Association, American Psychologic Association e o National Council on Measurement in Education - AERA, APA e NCME, (1999), publicaram o Standards for educational and psychological testing para testes psicológicos e educacionais de modo a nortear e sistematizar critérios e informações para a construção de instrumentos e prática da testagem.

No Brasil, destaque deve ser dado ao trabalho de Wechsler (1999) que elaborou um guia com orientações importantes sobre questões éticas no processo de avaliação psicológica. Ressalta-se também a ação do Conselho Federal de Psicologia (CFP), que publicou a Resolução $n^{\circ}$ 25/2001 relativa às normas de elaboração, comercialização 
e utilização dos testes psicológicos (CFP, 2001). Mais recentemente, a resolução foi substituída pela Resolução nº2/2003; ambas determinaram critérios mínimos que devem ser contemplados nos manuais dos instrumentos para comercialização, tais como definição do construto, fundamentação teórica da avaliação proposta, evidências de validações e precisão e a descrição do sistema de correção e interpretação a ser adotado.

Essas medidas do CFP, aliadas ao esforço dos pesquisadores da área de avaliação psicológica, apontam para a necessidade da divulgação de informações científicas que subsidiem uma prática profissional mais responsável e a elaboração de testes que busquem uma representação mais próxima possível do traço ou amostra de comportamento que se deseja investigar. Para isso, são necessários estudos que avaliam os chamados "parâmetros psicométricos" dos testes. Essas pesquisas planejam oferecer uma interpretação mais correta possível dos dados coletados, no devido contexto em que se pretende analisá-los, minimizando os possíveis prejuízos de uma avaliação equivocada. Nesse sentido é que este trabalho pretende contribuir, apresentando evidências de validade convergente de um teste de Inteligência Emocional, somando ao esforço de definição desse construto psicológico.

O final do século XX, mais especificamente a década de 90, registrou um crescente interesse em torno do conceito de Inteligência Emocional, que resultou em diversas publicações apresentadas por Mayer, Salovey e Caruso (2002). Essa tendência pode ser observada tanto em pesquisas que visavam a delimitação de construto, como em outras que investigavam aspectos de medição e comprovação empírica, baseados nos conceitos de inteligência a partir de um modelo psicométrico.

O termo Inteligência Emocional foi utilizado pela primeira vez no início da década de 90 por Salovey e Mayer (1990). É definido pelos autores como "a capacidade de perceber, avaliar e expressar emoções com precisão; a capacidade de acessar e/ou gerar sentimentos quando estes facilitam o pensamento; a capacidade de entender as emoções e o conhecimento emocional e a capacidade de regular emoções para promover o crescimento emocional e intelectual" (Mayer, Salovey \& Caruso, 1997, p. 10). O conceito foi mais amplamente divulgado na comunidade não científica por meio do livro de Daniel Goleman (1996), levando a muitos a impressão de tratar-se de uma idéia nova. Contudo, apesar do interesse em torno deste assunto ter sido intensificado mais recentemente, na verdade alguns conceitos que envolvem a Inteligência Emocional já se mostravam presentes em estudos ao longo de todo o século XX.

Desde E. L. Thorndike (apud Hedlund \& Sternberg, 2002), pesquisadores vêm propondo a existência da habilidade social como um componente da inteligência, apontando para modelos que vão além das habilidades intelectuais ou cognitivas, sobre as quais repousam a idéia de um fator $g$ (aptidão geral) e as medições de QI. Nestes novos modelos, encaixam-se, por exemplo, as chamadas inteligências não-cognitivas, que abrangem conceitos como in- teligências Emocional, Social e Prática (Hedlund \& Sternberg, 2002).

Thorndike (apud Hedlund \& Sternberg, 2002) propôs que a Inteligência Social abrangeria as habilidades de entender o outro e agir de maneira sábia em relação aos demais. Nesta época do início do século XX, aceitava-se empiricamente a idéia de que as inteligências estariam divididas em dois ou três subgrupos, sendo o primeiro formado pela inteligência chamada Verbal-Proposicional. Essa incluiria, por exemplo, extensão do vocabulário, fluência verbal e pensamento lógico. O segundo, denominado Inteligência de Organização Espacial, incluiria a capacidade de agrupar objetos, construir modelos e padrões. O terceiro subgrupo seria o da Inteligência Social, para a qual vários pesquisadores direcionaram esforços no intuito de conhecer e medir o construto. Houve, no entanto, sérias dificuldades neste sentido, que acabaram por desencorajar tais estudos. A principal delas foi a constatação de que a Inteligência Social se mostrava altamente relacionada às outras duas inteligências, a ponto de parecer impossível separá-la. As conclusões indicavam não se justificar a necessidade de investigação sob esta perspectiva. Assim, apesar da evidência de que vários dos testes construídos ao longo do século para medir habilidades verbais e espaciais abrangerem aspectos de raciocínio social, tais aspectos não eram avaliados como pertencentes a um domínio independente (Mayer \& Salovey, 1999).

A partir desse período do início do século XX, e apesar das dificuldades aparentes, vários pesquisadores desenvolveram teorias que vieram a questionar as visões tradicionais da inteligência fundamentadas no QI e em um fator único geral. Destaca-se a teoria das Inteligências Múltiplas de Gardner (1994) que incorporava o que ele chamou de Inteligência Interpessoal, definida como a capacidade de entender o outro e agir de acordo com esse entendimento, e a Inteligência Intrapessoal, como a habilidade de entender a si próprio. Já a chamada Inteligência Prática foi proposta pelo psicólogo americano Robert Sternberg et al. (2000) dentro de seu modelo triárquico. Esse modelo se divide em três subteorias denominadas componencial, experiencial e contextual, que abrangem aspectos e processos diferentes do funcionamento cognitivo interno do indivíduo e de sua relação com o meio externo. A inteligência prática se insere na subteoria contextual como uma habilidade exercida no cotidiano das pessoas e transmitida fora da educação formal, que envolve aspectos como demonstrar eficiência em saber o que dizer a alguém, da melhor maneira e no momento mais adequado. A exemplo da Inteligência Emocional, da forma como apresentada por Goleman (1996), a Inteligência Prática seria, supostamente, mais eficiente que a inteligência clássica (fator g) na predição do sucesso em várias áreas importantes da vida, inclusive do sucesso profissional. Não parece haver, no entanto, dados de pesquisas que corroborem tais postulados.

Outro modelo que merece destaque pelo relacionamento importante com os pressupostos teóricos da Inteligência Emocional, é a teoria Cattell-Horn-Carroll (CHC) de 
habilidades cognitivas. Desenvolvida a partir de uma integração dos modelos Gf-Gc de Horn e Cattell (1966) e os três estratos de Carroll (1993), é considerada atualmente a teoria de inteligência mais evoluída da abordagem psicométrica. Enfatiza as camadas I e II do modelo Gf-Gc. A camada I representa uma diversidade de habilidades cognitivas mais específicas que se agrupam em dez fatores mais amplos (II camada) (McGrew \& Flanagan, 1999). Dois desses fatores interessam particularmente a esta pesquisa. As inteligências Fluida (Gf), que envolve raciocínio para solucionar situações e problemas novos, e Cristalizada (Gc), que representa o processamento de conhecimentos e experiências previamente adquiridos (Primi \& Almeida, 2000).

Como uma das derivações teóricas dos modelos de inteligência não tradicionais, o campo da Inteligência Emocional tem-se caracterizado, ao longo de seu desenvolvimento histórico, pelas dificuldades de mensuração. Isso ocorre, em parte, devido aos problemas teóricos de delimitação do construto, ou mesmo por questões específicas sobre os tipos de instrumentos utilizados para medir essa forma de aptidão.

Dentre as dificuldades que comprometem o estabelecimento da Inteligência Emocional como um construto científico, pode-se ressaltar o fato de que, cada vez que o tema é discutido, o novo debate parece demandar uma definição distinta, assim como outras justificativas para sua importância. Esse quadro, ao contrário de facilitar um delineamento mais preciso e convergente do conceito, termina por levar a uma abrangência cada vez maior, e muitas vezes uma sobreposição com outros construtos.

Ainda na questão do esforço em relacionar a Inteligência Emocional ao campo das habilidades cognitivas, Schaie (2001), comentando o artigo de Roberts, Zeidner e Matthews (2001), sobre a definição da Inteligência Emocional, salienta a necessidade de pesquisas que investiguem evidências de validade convergente e discriminante, e que favoreçam o estabelecimento do construto, distinguindoo de traços de personalidade. Sugere ainda que tal investigação seja processada em adultos, tornando possível uma melhor identificação de tais diferenciações. A razão dessa sugestão pode estar relacionada ao fato de que, determinados aspectos da maturidade emocional, importantes para o desenvolvimento de habilidades que compõem o construto, estejam consolidadas somente no final da adolescência ou início da vida adulta. O que justifica a opção de universitários para composição da amostra.

Por essa razão é que esse trabalho foi proposto, e para tanto, elegeu o MSCEIT como o instrumento de pesquisa. Trata-se de um teste que avalia medidas de desempenho, apresentando tarefas que procuram dimensionar as habilidades do sujeito. Em específico, o presente estudo investigou, através de correlações, a validade de construto do MSCEIT no que tange à capacidade do teste avaliar diferentes aspectos da Inteligência Emocional, contemplados no instrumento e outros aspectos da inteligência co- bertos pelo BPR-5. A comparação entre dois testes permite investigar evidências de validade convergente, ou seja, o grau de concordância entre os construtos medidos (AERA, APA \& NCME, 1999).

\section{Método}

\section{Participantes}

Participaram do estudo 191 estudantes universitários de cursos diferentes das áreas de ciências humanas e exatas de um Centro Universitário particular do interior paulista. A amostra se dividiu em $40 \%$ de alunos do curso de Pedagogia ( $n=77)$; 26\% de Administração de Empresas $(n=50) ; 14 \%$ de Sistemas de Informação $(n=28) ; 11 \%$ do curso de Educação Física $(n=22)$; e $7 \%$ proveniente do curso de Ciências Contábeis $(n=14)$. Em relação ao semestre letivo, a amostra foi composta por alunos do primeiro $(61 \% ; n=117)$ e sétimo semestres $(39 \% ; n=74)$. Vale salientar que os cursos Ciências Contábeis e Sistemas de Informação ainda não possuem turmas de sétimo semestre por tratar-se de cursos novos, portanto, esses cursos foram representados apenas no grupo do primeiro semestre.

A idade dos sujeitos variou de 17 a 60 anos. Nove participantes não indicaram idade. A média das idades foi de 25 anos e o desvio padrão 7,9. Em relação ao sexo, a amostra incluiu $43 \%$ de participantes do sexo masculino $(n=78)$ e $57 \%$ do sexo feminino $(n=104)$.

\section{Instrumentos}

\section{Mayer-Salovey-Caruso Emotional Intelligence Test - MSCEIT}

Trata-se de um instrumento de medida de Inteligência Emocional preparado para avaliar duas áreas amplas: experiencial e estratégica. Estas áreas permitem avaliar quatro facetas do construto: percepção, facilitação do pensamento, compreensão e gerenciamento das emoções. O material inclui um caderno de respostas, com um total de 141 itens, na forma de medidas por desempenho que podem ser aplicados a sujeitos de, no mínimo, 17 anos de idade, sem limite máximo. Os itens são organizados em oito seções: Faces, Figuras, Facilitação, Sensações, Transição, Mistura, Administração de emoções e Relações emocionais.

Um dos aspectos deste instrumento que tem recebido mais críticas é seu sistema de pontuação e correção. O manual instrui que os dados sejam encaminhados para a editora nos EUA para correção, apesar da solicitação de adaptação do instrumento junto à editora.

O manual do MSCEIT descreve dados relativos à precisão baseados na amostra de padronização ( $N=5.000)$. Utilizando o método das metades (split-half), o teste apresentou índice geral de fidedignidade de 0,91. A análise da consistência interna para as áreas experiencial e estratégica foi igual a 0,90 e 0,85, respectivamente. Em relação aos 
estudos de validade apresentados, o manual esclarece que os dados se referem a pesquisas realizadas com a versão anterior do instrumento, o MSCEIT 1.1, e justifica esse procedimento a partir da similaridade entre ambos. Diversas evidências de validade são apresentadas pelos autores no instrumento original. Quanto à validade discriminante, os autores relatam correlações moderadas com testes de QI, na ordem de $r=0,36$ e $r=0,38$ para amostras de 503 e 239 sujeitos respectivamente. Conforme o modelo teórico proposto, isso indica, ao mesmo tempo, certo grau de convergência da IE com as demais inteligências, e uma independência que reforça a idéia de tratarse de um construto à parte.

\section{Bateria de Provas de Raciocínio - BPR-5}

Trata-se de um instrumento que avalia habilidades cognitivas (Inteligência Fluida e cristalizada). Organizado nos formatos A e $\mathrm{B}$, destina-se à aplicação em alunos de sexta à oitava série do ensino fundamental e alunos do ensino médio, respectivamente. Possui cinco provas, da forma como segue: raciocínio abstrato (RA), que avalia basicamente a Inteligência Fluida (Gf), definida como a capacidade de raciocinar em situações novas, criar conceitos e compreender implicações; raciocínio verbal (RV), que avalia tanto a Inteligência Fluida (Gf), quanto a Cristalizada (Gc), definida por sua vez, como a extensão e profundidade do conhecimento verbal vocabular e a capacidade de raciocinar utilizando conceitos previamente aprendidos; raciocínio espacial (RE), cujo objetivo é avaliar em parte a Inteligência Fluida (Gf), mas principalmente a capacidade de Processamento Visual (Gv), definida como a habilidade de representar e manipular imagens mentais; raciocínio numérico $(\mathrm{RN})$, que mede a Inteligência Fluida (Gf) e a Habilidade Quantitativa (Gq), definida como a compreensão de conceitos quantitativos básicos, como soma, subtração, multiplicação e divisão e manipulação de símbolos numéricos; e raciocínio mecânico (RM), que avalia a Inteligência Fluida (Gf) e também conhecimentos práticos mecânicos.

O manual do BPR-5 apresenta estudos de validade e precisão do instrumento com amostras de sujeitos brasileiros e portugueses. O primeiro estudo avaliou 1.243 sujeitos dos dois países, aplicando as formas A e B do teste em alunos do ensino fundamental e médio. A precisão foi investigada por meio do método das metades (Split-half) e as correlações variaram de 0,65 a 0,87. Também foi pesquisada a consistência interna com base na correlação de Pearson (Alfa I) e na correlação tetracórica. Quanto às evidências de validade, foi aplicada uma Análise Fatorial da matriz de correlação entre os cinco subtestes para investigar se as diferentes provas relacionavam-se ao mesmo fator, no caso, Inteligência Fluida (Gf). O resultado apontou para um único fator responsável por pouco mais de $50 \%$ da variância dos escores, confirmando o esperado. Foram estabelecidas correlações entre o BPR-5 e o desempenho de alunos, medido por meio de suas notas escolares e a consistência interna apontou coeficientes de correlação tetracórica (Alfa II) que variaram de 0,75 a 0,94.

\section{Procedimentos}

Após aprovação do projeto pelo Comitê de Ética, os dados foram obtidos por meio da aplicação coletiva dos instrumentos. Foram utilizadas duas sessões (uma para o BPR-5 e outra para o MSCEIT). Os testes foram aplicados após a concessão do Termo de Consentimento Livre e Esclarecido (TCLE) dos participantes. Para a aplicação do BPR-5 foi respeitado a ordem indicada no manual (RV, RA, RM, RE e RN) e adotado um intervalo para descanso de aproximadamente 15 a 20 minutos entre as três primeiras e as duas últimas provas.

\section{Resultados}

O trabalho buscou evidência de validade convergente do construto Inteligência Emocional, comparando o resultado do MSCEIT com a Inteligência fluida e cristalizada, mais especificamente com os cinco tipos de raciocínio avaliados pelo BPR-5. Por razões didáticas, optou-se por dividir a apresentação dos resultados em duas partes. A primeira apresenta os dados da estatística descritiva de cada instrumento administrado. A segunda, apresenta inicialmente as correlações entre as sub-escalas do MSCEIT e, em seguida, as correlações entre os instrumentos.

A Tabela 1 apresenta os resultados da análise descritiva do BPR-5. Verificou-se que a maior média foi obtida na prova de Raciocínio verbal $(M=15,57)$ contra a de Raciocínio espacial, que aparece com a menor média $(M=9,72)$.

Tabela 1

Estatística Descritiva do BPR-5

\begin{tabular}{llllll}
\hline & N & Média & DP & Mínimo & Máximo \\
\hline Raciocínio abstrato & 191 & 14,85 & 4,164 & 0 & 22 \\
Raciocínio verbal & 191 & 15,57 & 3,723 & 2 & 23 \\
Raciocínio mecânico & 191 & 10,09 & 4,501 & 0 & 22 \\
Raciocínio espacial & 191 & 9,72 & 4,551 & 0 & 20 \\
Raciocínio numérico & 191 & 10,39 & 4,064 & 2 & 20 \\
Escore geral & 191 & 60,64 & 15,723 & 20 & 95 \\
\hline
\end{tabular}


O maior desvio padrão foi encontrado em Raciocínio espacial $(D P=4,551)$, e o menor em Raciocínio verbal $(D P=3,723)$. A pontuação máxima foi em Raciocínio verbal, atingindo 23 dos 25 pontos possíveis. As provas de Raciocínio abstrato, mecânico e espacial, apresentaram a pontuação mínima de o pontos.

Foram realizadas duas análises descritivas do MSCEIT, a primeira relacionando sub-testes e a segunda, relacionado as facetas. Tais análises podem ser visualizadas na Tabela 2 . A maior média foi constatada no sub-teste Transição $(M=41,44)$ e a menor na de Relações $(M=33,91)$.
Uma questão a ser considerada quanto à maior média em Transição é a diferença do tipo de resposta desse subteste (múltipla escolha) em relação aos demais (escalas Likert). Isso pode ter influência na facilidade com que o sujeito reconhece a resposta do item. O maior desvio padrão ocorreu no sub-teste Figuras $(D P=10,421)$, e o menor em Transição $(D P=6,789)$. A pontuação mínima foi encontrada no sub-teste Figuras $(7,37)$ e a máxima em Facilitação $(54,84)$.

Tabela 2

Estatística Descritiva dos Sub-Testes, Áreas e Facetas do MSCEIT

\begin{tabular}{llllll}
\hline & N & Média & DP & Mínimo & Máximo \\
\hline Faces & 191 & 38,79 & 8,442 & 11,23 & 52,53 \\
Figuras & 191 & 36,82 & 10,421 & 7,37 & 51,99 \\
Facilitação & 189 & 41,21 & 8,332 & 15,90 & 54,84 \\
Sensação & 190 & 34,87 & 8,295 & 13,68 & 49,65 \\
Transição & 191 & 41,44 & 6,783 & 11,89 & 54,58 \\
Mistura & 190 & 36,02 & 7,612 & 9,01 & 49,54 \\
Administração & 188 & 36,20 & 9,605 & 11,62 & 52,50 \\
Relações & 189 & 33,91 & 9,624 & 11,74 & 50,38 \\
& & & & 17,18 & 51,25 \\
Percepção emoções & 191 & 37,81 & 7,641 & 17,17 & 50,11 \\
Facilitação o pensamento & 190 & 38,02 & 6,873 & 14,47 & 50,35 \\
Compreensão de emoções & 191 & 38,69 & 6,043 & 12,14 & 51,32 \\
Gerenciamento de emoções & 191 & 34,98 & 8,414 & 19,81 & 49,51 \\
Área experiencial & 191 & 37,87 & 6,301 & 15,94 & 48,13 \\
Área estratégica & 191 & 36,83 & 5,962 & 22,04 & 46,66 \\
\hline IE Total & 184 & 37,52 & 5,142 & & \\
\hline
\end{tabular}

No que diz respeito às facetas do MSCEIT, a estatística descritiva indicou que a maior média ocorreu em Compreensão de emoções $(M=38,69)$ e a menor em Gerenciamento de emoções $(M=34,98)$. Nessa última faceta, ainda ocorreu o maior desvio padrão $(D P=8,414)$ e os valores mínimos $(12,14)$ e máximo $(51,32)$. A Tabela 2 também apresenta os dados referentes às duas áreas amplas e ao total da IE.

As correlações de Pearson obtidas entre as escalas do MSCEIT e entre os instrumentos MSCEIT e BPR-5 estão apresentadas nas Tabelas 3, 4, 5 e 6 .
Todos os sub-testes do MSCEIT apresentaram correlações positivas e significativas, com exceção dos sub-testes Faces e Mistura $(r=0,122, \mathrm{p}=0,10)$. As correlações mais baixas ocorreram entre os sub-testes Mistura e Figuras ( $\left.r=0,171^{*}\right)$, Administração e Mistura ( $\left.r=0,171^{*}\right)$, Transição e Facilitação ( $\left.r=0,193^{*}\right)$, Relações e Mistura ( $\left.r=0,191^{*} *\right)$, Relações e Faces $\left(r=0,208^{*} *\right)$ e Transição e Faces $\left(r=0,205^{*} *\right)$. A pesquisa de Mayer, Salovey, Caruso e Sitarenios (2003) também apresentou baixas correlações entre os sub-testes Faces e Mistura $\left(r=0,17^{*} *\right)$ e Faces e Relações $\left(r=0,19^{* * *}\right)$. 
Jesus Junior, A. G. \& Noronha, A.P. P. (2007). Inteligência Emocional e Provas de Raciocínio: Um Estudo Correlacional.

Tabela 3

Correlações entre os Sub-Testes do MSCEIT

\begin{tabular}{|c|c|c|c|c|c|c|c|c|}
\hline & Faces & Figuras & Facilitação & Sensação & Transição & Mistura & Administração & Relações \\
\hline Figuras & $r$ & $0,302^{\text {*⿻丷木 }}$ & - & & & & & \\
\hline Facilitação & $r$ & $0,402^{* *} *$ & $0,283^{*} * *$ & - & & & & \\
\hline Sensação & $r$ & $0,303^{*} *$ & 0,311 米米 & $0,374^{*} * *$ & - & & & \\
\hline Transição & $r$ & $0,205^{* *}$ & $0,244^{* * *}$ & $0,193^{*}$ & $0,273^{* * *}$ & - & & \\
\hline Mistura & $r$ & 0,122 & $0,171^{*}$ & 0,201 米米 & $0,294^{* * *}$ & $0,385^{* * *}$ & - & \\
\hline Administração & $r$ & $0,244^{*}$ *⿻丷木 & $0,274^{*} * *$ & $0,372^{* * *}$ & $0,322^{* * *}$ & $0,313^{*} *$ & $0,171^{*}$ & - \\
\hline Relações & $r$ & $0,203^{*} *$ * & $0,301^{* * *}$ & $0,213^{*} *$ * & $0,355^{*}$ 米 & $0,272^{\text {*** }}$ & $0,191^{\text {**** }}$ & 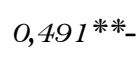 \\
\hline
\end{tabular}

Notas. ${ }^{*}$ Coeficientes com diferença significativa de zero $(p<0,05)$; ** Coeficientes com diferença muito significativa de zero $(p<0,01)$.

Corroborando a proposta do MSCEIT de avaliar as quatro facetas da IE (Percepção, Facilitação, Compreensão e Gerenciamento) por meio de dois sub-testes cada, foi possível perceber que as maiores correlações deram-se entre os sub-testes que avaliam a mesma faceta: Faces e Figuras $\left(r=0,306^{* *}\right)$, Facilitação e Sensação $\left(r=0,374^{* * *}\right)$, Transição e Mistura ( $r=0,385^{*}$ *) e Administração e Relações $\left(r=0,49^{* *}\right)$, sendo essa última a mais alta. A única exceção foi a alta correlação encontrada entre os sub-testes Faces e Facilitação $\left(r=0,401^{*} *\right.$, representantes de facetas distintas. Esses dados indicam razoável comunalidade dos diversos sub-testes em relação à faceta que pretende avaliar. Uma pesquisa com amostra americana $(N=1.985)$ ratifica esses dados, e mostra que as maiores correlações ocorreram entre os sub-testes das facetas Compreensão $(r=0,59)$ e Gerenciamento $(r=0,58)$ (Mayer et al., 2003).

Tabela 4

Correlações entre Facetas e Sub-Testes do MSCEIT

\begin{tabular}{|c|c|c|c|c|c|c|c|c|c|}
\hline & & 导 & 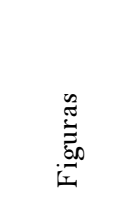 & 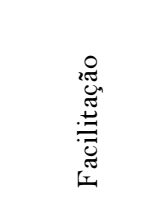 & 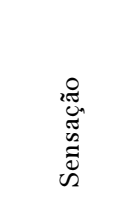 & 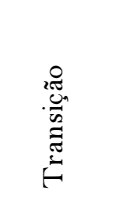 & 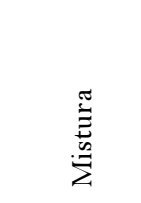 & 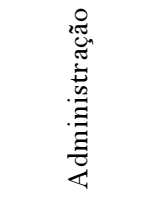 & 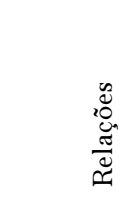 \\
\hline Percepção & $r$ & $0,762^{* * *}$ & $0,853^{* * *}$ & $0,414^{* * *}$ & O,383 *** & $0,272^{*} *$ & $0,183^{*}$ & $0,325^{* * *}$ & O,314**⿻丷木 \\
\hline Facilitação & $r$ & $0,421^{*} *$ & 0,351 *⿻丷木 & $0,832^{*} *$ * & 0,834 *** & $0,272^{*} *$ & 0,306 米米 & $0,422^{*} *$ * & $0,3411^{*} *$ \\
\hline Compreensão & $r$ & $0,204^{* * *}$ & 0,241 米米 & $0,252^{*} *$ * & 0,331 *** & $0,812^{* * *}$ & $0,861^{* * *}$ & $0,303^{* * *}$ & $0,282^{*} *$ \\
\hline Gerenciamento & $r$ & $0,264^{* * *}$ & O,332 *** & O,353米米 & O,394*** & $0,351^{* * *}$ & $0,211^{* * *}$ & $0,873^{* * *}$ & $0,874^{*}$ *⿻丷木 \\
\hline
\end{tabular}

Notas. *Coeficientes com diferença significativa de zero $(p<0,05) ;{ }^{*}$ * Coeficientes com diferença muito significativa de zero $(p<0,01)$.

As correlações entre o MSCEIT e o BPR-5 foram descritas nas Tabelas 5 e 6. A Tabela 5 apresenta as correlações entre os oito sub-testes do MSCEIT e as cinco provas do BPR-5. A Tabela 6 apresenta as correlações entre as quatro facetas e as duas áreas do MSCEIT e as cinco provas do BPR-5.
Raciocínio abstrato, que avalia basicamente a Inteligência Fluida (Gf), se correlacionou de maneira positiva e significativa com Faces $\left(r=0,291^{* *}\right)$, Figuras $\left(r=0,307^{* * *}\right)$, Transição ( $\left.r=0,213^{* *}\right)$ e Mistura ( $\left.r=0,254^{* *}\right)$. Raciocínio verbal foi o que apresentou maior número de correlações positivas e significativas, com Faces $\left(r=0,210^{* *}\right)$, Figuras ( $\left.r=0,222^{*} *\right)$, Sensação $\left(r=0,155^{*}\right)$, Transição $\left(r=0,235^{*} *\right)$, Mistura $\left(r=0,211^{* *}\right)$ e Administração $\left(r=0,175^{*}\right)$.

Tabela 5

Correlações entre BPR-5 e Sub-Testes do MSCEIT

\begin{tabular}{llllllllll}
\hline & & Faces & Figuras & Facilitação & Sensação & Transição & Mistura & Administração Relações \\
\hline Raciocínio abstrato & $r$ & $0,291^{* *}$ & $0,307 * *$ & 0,103 & 0,072 & $0,213^{* *}$ & $0,254 * *$ & 0,134 & 0,121 \\
Raciocínio verbal & $r$ & $0,210^{* *}$ & $0,222^{* *}$ & 0,123 & $0,155^{*}$ & $0,235^{* *}$ & $0,211^{* *}$ & $0,175^{*}$ & 0,141 \\
Raciocínio mecânico & $r$ & 0,010 & $-0,071$ & $-0,115$ & $-0,060$ & $-0,053$ & 0,075 & $-0,060$ & $-0,055$ \\
Raciocínio espacial & $r$ & 0,082 & 0,081 & 0,033 & $-0,012$ & 0,110 & $0,202^{* *}$ & 0,041 & 0,084 \\
Raciocínio numérico & $r$ & 0,133 & 0,105 & 0,095 & 0,079 & $0,224 * *$ & $0,162^{*}$ & $0,162 *$ & 0,082 \\
\hline
\end{tabular}

Notas. *Coeficientes com diferença significativa de zero $(p<0,05) ; *$ * Coeficientes com diferença muito significativa de zero $(p<0,01)$. 
Raciocínio mecânico, não se correlacionou significativamente com nenhum dos sub-testes do MSCEIT, apresentando inclusive seis correlações negativas do total de oito. Raciocínio espacial, correlacionou-se de forma positiva e significativa apenas com o sub-teste Mistura $\left(r=0,209^{*}\right)$. Finalmente, Raciocínio numérico, apresentou correlação apenas com Transição $\left(r=0,224^{*} *\right.$, Mistura $\left(r=0,162^{*}\right)$ e Administração $\left(r=0,163^{*}\right)$.

Em relação às áreas e facetas do MSCEIT e o BPR-5 houve grande número de correlações positivas e significativas. Como pode-se observar na Tabela 6, Raciocínio abstrato só não se correlacionou com Facilitação, que corresponde ao aspecto da IE que indica a habilidade de utilizar as emoções para guiar os pensamentos e escolhas. Provavelmente isso seja devido ao fato de que os itens do sub-teste Facilitação sejam de caráter extremamente práticos (por exemplo: que tipo de estado de humor pode ajudar a criar uma decoração nova, excitante para uma festa de aniversário?) e não tão abstratos. Raciocínio verbal apresentou correlações positivas e significativas com todas as áreas e facetas. Raciocínio mecânico, novamente não se correlacionou significativamente com nenhuma das áreas do MSCEIT, apresentando inclusive todas as correlações negativas com exceção de Compreensão. Raciocínio espacial se correlacionou apenas com a faceta Compreensão ( $\left.r=0,182^{*}\right)$ e sua respectiva área estratégica $\left(r=0,142^{*}\right)$. Raciocínio numérico só não apresentou correlação significativa com as facetas Percepção e Facilitação.

Foi possível ainda verificar que o Escore Geral do BPR5 correlacionou-se com as facetas Percepção $\left(r=0,217^{*} *\right)$ e Compreensão ( $r=0,261^{*}$ * $)$, e com as áreas Experiencial $\left(r=0,174^{*}\right)$ e Estratégica $\left(r=0,230^{*} *\right)$. A IE, de forma geral, correlacionou-se com três áreas do BPR-5. Raciocínio abstrato $\left(r=0,251^{*} *\right)$, Raciocínio verbal $\left(r=0,280^{*} *\right)$ e Raciocínio numérico $\left(r=0,171^{*}\right)$. Esses resultados confirmam os apontamentos de Schaie (2001) de que deveria ser esperada uma maior correlação da IE com a Inteligência Cristalizada, portanto, com o Raciocínio verbal, e uma correlação mais moderada com a Inteligência Fluida, no caso o Raciocínio abstrato. Finalmente a correlação entre a IE e o Escore geral do BPR-5 apresentou-se positiva, baixa e significativa $(r=0,184 *$.

Tabela 6

Correlações entre BPR-5, Áreas e Facetas do MSCEIT

\begin{tabular}{|c|c|c|c|c|c|c|c|c|}
\hline & & 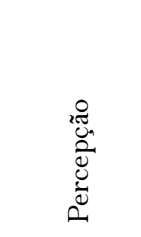 & 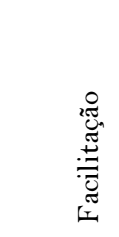 & 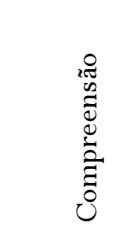 & 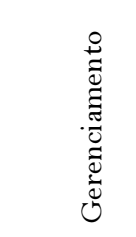 & 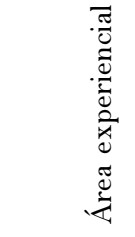 & 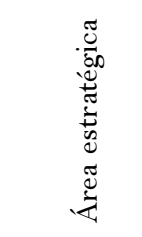 & 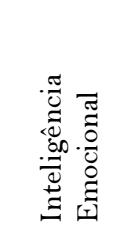 \\
\hline Raciocínio abstrato & $r$ & $0,363^{* * *}$ & 0,106 & $0,292^{* * *}$ & $0,170^{*}$ & $0,293^{* * *}$ & $0,265^{* * *}$ & $0,251^{\text {*** }}$ \\
\hline Raciocínio verbal & r & $0,263^{* *}$ & $0,164^{*}$ & $0,261^{* * *}$ & $0,195^{*}$ *⿻丷木 & $0,252^{* * *}$ & $0,271^{*}$ *⿻丷木 & $0,280^{* * *}$ \\
\hline Raciocínio mecânico & $r$ & $-0,045$ & $-0,115$ & $\mathrm{O}, \mathrm{O} 2 \mathrm{O}$ & $-0,055$ & $-0,088$ & $-0,035$ & $-0,106$ \\
\hline Raciocínio espacial & $r$ & 0,101 & 0,012 & $0,182^{*}$ & 0,075 & $\mathrm{O}, \mathrm{O} 72$ & $0,142^{*}$ & 0,111 \\
\hline Raciocínio numérico & $r$ & 0,140 & 0,101 & $0,244^{*}$ *⿻丷木 & $0,153^{*}$ & $0,152^{*}$ & $0,233^{*} *$ & $0,171^{*}$ \\
\hline Escore geral & $r$ & $0,211^{\text {*⿻丷木 }}$ & 0,063 & $0,261^{\text {*⿻丷木 }}$ & 0,141 & $0,174^{*}$ & $0,230^{*}$ *⿻丷木 & $0,184^{*}$ \\
\hline
\end{tabular}

Notas. ${ }^{*}$ Coeficientes com diferença significativa de zero $(p<0,05)$; ** Coeficientes com diferença muito significativa de zero $(p<0,01)$.

\section{Discussão e Conclusões}

A análise descritiva do BPR-5 apresenta média de acertos mais alta na prova de Raciocínio verbal. Esse dado revela que os sujeitos da amostra estudada, em geral, tiveram maior facilidade em resolver tarefas envolvendo habilidades relacionadas às palavras e seus significados. Visto que a maioria dos sujeitos era proveniente da área de humanas, este resultado condiz com o esperado, tanto quanto o fato de as piores médias terem sido encontradas em tarefas que exigem raciocínios em relação a conteúdos concretos, como espacial e mecânico. Essa última tendência também foi constatada no estudo apresentado no manual do BPR-5, realizado com uma amostra de 411 alunos portugueses do curso correspondente ao ensino médio brasileiro. Entre os portugueses, os escores $\mathrm{Z}$ dos alunos da área de Humanas mostraram-se bem abaixo da média das demais áreas (Científica-natural, Artes, Econômicasocial) nas provas RE e RM (Primi \& Almeida, 2000).

Outra possibilidade a ser considerada é o fato de as duas primeiras provas aplicadas (RV e RA) terem sido as que apresentaram melhores desempenhos devido à menor influência do fator cansaço.

As correlações entre as facetas e os sub-testes do MSCEIT confirmaram a adequação dos dados à proposta do instrumento, considerando que se observaram altas correlações positivas entre os sub-testes e suas respectivas facetas que indicam quatro aspectos do construto da IE, conforme apresentado na Tabela 4 (Mayer, Salovey \& Caruso, 1999).

Os dados apresentaram maior número de correlações das escalas do MSCEIT em relação com o Raciocínio ver- 
bal. Uma justificativa possível para tal resultado estaria vinculada ao fato de a maioria da amostra ter sido composta por sujeitos da área de Ciências Humanas, e que indivíduos que optam por essa área de estudos, tenderiam a apresentar habilidades verbais mais refinadas, o que lhes proporcionaria melhor comunicação e interação com os outros facilitando o acesso, identificação e administração das emoções.

Segundo Hedlund e Sternberg (2002), desde a época dos estudos sobre a Inteligência Social proposta por Thorndike e uma das precursoras da IE, as pesquisas apontavam para certa sobreposição entre a inteligência chamada VerbalProposicional que incluía habilidades verbais e pensamento lógico e a Inteligência Social. Isso de certa forma é esperado pelo fato de a expressão verbal ser de fundamental importância para a interação com os outros e, conseqüentemente o manejo de conteúdo emocional, o que justificaria o maior número de correlações dos sub-testes do MSCEIT com Raciocínio verbal e abstrato. Segundo a visão do modelo do início do século XX, em oposição, estaria a inteligência de organização espacial, abrangendo os raciocínios mecânico e espacial. Esses últimos envolveriam menos pensamento lógico e mais capacidade de manipular mentalmente objetos e fenômenos concretos.

Mayer e Salovey (1999) advogam que a IE envolveria o raciocínio lógico e abstrato sobre informações de conteúdo emocional. Essa análise justifica o fato dessas duas provas do BPR-5, Raciocínio mecânico e Raciocínio espacial, praticamente não apresentarem correlações com os subtestes do MSCEIT. De acordo com a fundamentação teórica contida no manual do BPR-5, a prova de Raciocínio abstrato avalia a Inteligência Fluida (Gf), enquanto que a de Raciocínio Verbal avalia a Inteligência Cristalizada (Gc).

O manual do BPR-5 (Primi \& Almeida, 2000) define a Inteligência Fluida como «habilidade de raciocínio em situações novas, minimamente dependente de conhecimentos adquiridos» (p. 13) e Inteligência Cristalizada como «habilidade associada à extensão e profundidade dos conhecimentos adquiridos de uma determinada cultura» (p. 13). Admitindo-se que a IE envolve conhecimento adquirido sobre emoções, de natureza cultural (Gc), e raciocínio sobre problemas novos de natureza abstrata, podemos inferir que as provas de Raciocínio Verbal e Raciocínio Abstrato do BPR-5, por avaliar esses dois tipos de raciocínio estariam mais intimamente relacionadas com os diversos sub-testes do MSCEIT. Por outro lado, as provas de Raciocínio mecânico e Raciocínio espacial, guardam certa relação com a Inteligência Fluida (Gf), porém se aproximam mais fortemente de outros tipos de inteligência, no caso a que envolve conhecimentos práticos mecânicos e a de processamento visual, respectivamente.

A correlação entre a IE e o Escore geral do BPR-5 é um dado que merece ser salientado, já que tem relação direta com a hipótese que se pretendeu investigar nesse trabalho de pesquisa: de que o MSCEIT e o BPR-5 deveriam apresentar correlação baixa e significativa, indicando que a IE pode ser considerada um tipo de inteligência, porém distinto da inteligência clássica ou acadêmica.
Esperava-se que a correlação entre a IE e o escore geral do BPR-5 ( $\left.r=0,184^{*}\right)$ se apresentasse ligeiramente mais alta, para indicar mais claramente evidência de validade convergente. Vale ressaltar, no entanto, que segundo o modelo de inteligência de três estratos (Carroll, 1993), mais reconhecido atualmente, as habilidades cognitivas guardam diferenças em termos de suas correlações com o fator geral. Nesse modelo, Carrol demonstrou que a Inteligência Fluida (Gf) e Cristalizada (Gc) são as que respondem por maior influência sobre esse fator geral. Como já foi discutido nesse estudo, tanto o MSCEIT, quanto o BPR5 avaliam habilidades relacionadas a esses dois tipos de inteligência. No próprio manual do BPR-5 (Primi \& Almeida, 2000), encontramos a indicação de que todas as suas provas abrangem, em diferentes graus, a Inteligência Fluida.

Vale ressaltar ainda que, algumas provas, especificamente raciocínio mecânico (RM) e espacial (RE), representam com maior peso habilidades distintas da Inteligência Fluida. Raciocínio Mecânico relaciona-se mais com habilidades práticas mecânicas e Raciocínio Espacial, com processamento visual (Primi \& Almeida, 2000). Devido ao fato da IE, por conceito, integrar habilidades basicamente relacionadas às inteligências Fluida e Cristalizada, podemos hipotetizar que as correlações não significativas encontradas entre as provas RM e RE, e as áreas e facetas do MSCEIT, tenham influenciado a correlação entre IE e o BPR-5 como um todo, a ponto de reduzi-la ao índice abaixo do esperado. Partindo-se dessa hipótese, for am subtraídos os escores das provas RM e RE do escore geral do BPR-5, com o objetivo de investigar se essa alteração produziria uma correlação ligeiramente mais elevada, o que de fato aconteceu ( $r=0,281^{*}$ ), reforçando a evidência de validade convergente do MSCEIT. Corroborando essas considerações, o trabalho de Cobêro (2004) analisou uma amostra de 117 sujeitos e encontrou correlações mais altas entre o MSCEIT e o BPR-5 ( $r=0,36^{*} *$ ). Nesse caso, a prova RM, praticamente não se correlacionou com o MSCEIT. No entanto, a RE apresentou quase todas as correlações significativas.

A hipótese investigada nesse estudo supôs que os resultados encontrados na aplicação do Mayer, Salovey e Caruso, Emotional Intelligence Test-MSCEIT, se correlacionam com os resultados das Baterias de Provas de Raciocínio-BPR-5, de maneira significativa, mas em níveis baixo ou moderado. Essa evidência, corroborada no estudo indica que a IE é um construto independente da inteligência clássica ou do raciocínio. Ao mesmo tempo, uma correlação com tal perfil indicaria uma afinidade suficiente com o campo das habilidades cognitivas que permita contribuir com uma delimitação do construto que o insira no rol das inteligências.

Em vista disso, a presente pesquisa objetivou investigar a validade de construto do MSCEIT, comparando-o com o BPR-5. A intenção, ao se comparar os dois instrumentos, foi buscar evidências de validade convergente, ou seja, a capacidade do MSCEIT representar a IE como um construto que se correlaciona positiva e moderadamente 
com a Inteligência fluida, por tratarem-se ambos de habilidades cognitivas.

É importante, também, contextualizar os objetivos dessa pesquisa com a situação atual da área de avaliação psicológica, ressaltando assim a relevância dos resultados. A testagem psicológica, apesar de não ser uma atividade nova, tem vivido um momento de grande interesse e retomada no esforço de pesquisa, buscando aprimorar suas técnicas, tanto no âmbito da Psicologia no Brasil, quanto internacionalmente (Noronha \& Alchieri, 2002). Entretanto, quanto à construção, aprimoramento e pesquisas que contribuam para a melhoria da qualidade de instrumentos de avaliação psicológica, o Brasil ainda se encontra em situação bastante desfavorável. Esse panorama, no entanto, vem se alterando e já são notórias as mudanças que contribuem para o avanço dessa área nesse país.

Vale ressaltar que os dados discutidos nesse trabalho, somado aos demais que buscam um tratamento adequado e um modelo teórico sólido para o construto da IE, indicam para um campo de estudos promissor, porém ainda carente de subsídios empíricos. Pelo menos se comparado a outros construtos já estabelecidos da Psicologia. Isso deveria levar à cautela e senso crítico ao tratar-se de conclusões e afirmações, às vezes demasiadamente contundentes, relacionadas à IE, como infelizmente é comum na literatura não científica.

O estudo das correlações entre os sub-testes do MSCEIT e entre suas facetas nesse artigo, pretende investigar se o teste apresenta consonância com o construto da IE. As correlações situaram-se, em sua maioria, em um nível acima de $r=0,30$, confirmando o resultado encontrado no estudo norte-americano (Mayer et al., 2003). Conforme esperado, as correlações entre os pares de sub-testes que derivam de cada uma das quatro facetas, apresentaram as maiores correlações, acima de $r=0,80$, e a única correlação não significativa ocorreu entre os sub-testes Faces e Mistura.

A correlação entre o MSCEIT e o BPR-5 mostrou-se, no geral, baixa e significativa $(r=0,184, p<0,05)$. A hipótese, a priori, era de que os dois instrumentos deveriam apresentar correlação baixa a moderada e significativa, visto tratar-se de construtos distintos, porém, ambos representando um mesmo tipo de inteligência. O resultado corrobora a evidência de validade convergente do MSCEIT. A maioria das correlações significativas entre seus sub-testes e as cinco provas do BPR-5, observou-se nas provas de Raciocínio verbal (RV), Raciocínio abstrato (RA) e Raciocínio numérico ( $\mathrm{RN}$ ). Raciocínio espacial (RE) correlacionou-se apenas com o sub-teste Mistura e Raciocínio mecânico (RM) não apresentou correlação com nenhum dos sub-testes. Todas as quatro facetas do MSCEIT correlacionaram-se significativamente com a prova RV, três delas correlacionaram-se significativamente com RA (exceto Facilitação), duas com RN e somente uma faceta (Compreensão) correlacionou-se com RE. Em contrapartida, RM não apresentou correlação com nenhuma das áreas da IE. Justifica-se esse resultado pelo fato da maioria da amostra ser composta de estudantes da área de
Ciências Humanas e uma pequena parcela de Exatas, o que denota um desempenho mais harmônico entre IE, RV e RA. Por outro lado, esse resultado ajusta-se ao modelo teórico que pressupõe que a IE apresenta maior correlação com a Inteligência Cristalizada e Fluida, medidas mais especificamente pelas provas RV e RA, respectivamente.

Investigar as correlações entre o MSCEIT e o BPR-5, explorando evidências de validade convergente com a inteligência, foram objetivos razoavelmente alcançados. Porém, faz-se necessário que novas pesquisas sejam realizadas e novos dados sejam acrescentados para suprir as deficiências apontadas e permitir o avanço do estabelecimento da Inteligência Emocional como um construto sólido e científico.

\section{Referências}

American Educational Research Association, American Psychology Association \& National Council on Measurement in Education. (1999). Standards for Educational and Psychological Testing. Washington, DC: American Educational Research Association.

Anastasi, A., \& Urbina, S. (2000). Testagem Psicológica. Porto Alegre, RS: Artes Médicas.

Carroll, J. B. (1993). Human cognitive abilities: A survey of factoranalytic studies. New York: Cambridge University Press.

Cobêro, C. (2004). Inteligência Emocional: Validade do MSCEIT no contexto organizacional. Dissertação de Mestrado nãopublicada, Curso de Pós-Graduação em Psicologia, Universidade São Francisco, Itatiba, SP.

Conselho Federal de Psicologia. (2001). Resolução no 25/2001 [Versão eletrônica]. Retirado em 20 jan. 2004, de http:// www.pol.org.br

Conselho Federal de Psicologia. (2003). Resolução 02/2003 [Versão eletrônica]. Retirado em 20 jan. 2004, de http:// www.pol.org.br

Gardner, H. (1994). Estruturas da mente: A teoria das inteligências múltiplas. Porto Alegre, RS: Artmed.

Goleman, D. (1996). Inteligência emocional: A teoria revolucionária que redefine o que é ser inteligente. Rio de Janeiro, RJ: Objetiva.

Hedlund, J., \& Sternberg, R. J. (2002). Inteligências em excesso? Integrando as inteligências social, emocional e prática. In R. Bar-on \& J. Parker (Eds.), Manual de inteligência emocional: Teoria e aplicação em casa, na escola e no trabalho (pp. 111-131). Porto Alegre, RS: ArtMed

Horn, J. L., \& Cattell, R. B. (1966). Refinement and test of the theory of fluid and crystallized general intelligences. Journal of Educational Psychology, 57(5), 253-270.

International Test Comission. (2001). Diretrices internacionales para el uso de los tests [On-line]. Retirado em 20 jan. 2004, de http://www.cop.es/tests/Diretrices.htm

Mayer, J. D., \& Salovey, P. (1999). O que é inteligência emocional? In P. Salovey \& D. J. Sluyter (Eds.), Inteligência emocional na criança: Aplicações na educação e no dia-a-dia (pp. 15-49). Rio de Janeiro, RJ: Campus.

Mayer, J. D., Salovey, P., \& Caruso, D. R. (1997). Emotional IQ test [CD-ROOM]. Needham, MA: Virtual Knowledge.

Mayer, J. D., Salovey, P., \& Caruso, D. R. (1999). MSCEIT Item Booklet [Research Version 1.1.]. Toronto, Canada: MultiHealth Systems. 
Mayer, J. D., Salovey, P., \& Caruso, D. R. (2002). Inteligência emocional como Zeitgeist, como personalidade e como aptidão mental. In R. Bar-on \& J. Parker (Eds.), Manual de inteligência emocional: Teoria e aplicação em casa, na escola e no trabalho (pp. 81-97). Porto Alegre, RS: ArtMed.

Mayer, J. D., Salovey, P., Caruso, D. R., \& Sitarenios, G. (2003). Measuring emotional intelligence with the MSCEIT V2.0. Emotion, 3(1), 97-105.

McGrew, K. S., \& Flanagan, D. P. (1999). Introduction to the CH-C Theory. Institute for Applied Psychometrics. Retirado em 20 out. 2003, de http://www.iapsych.com

Noronha, A. P. P. (2002). Os problemas mais graves e mais frequientes no uso dos testes psicológicos. Psicologia: Reflexão e Crítica, 15(1), 135-142.

Noronha, A. P. P. \& Alchieri, J. C. (2002). Reflexões sobre os instrumentos de avaliação psicológica. In R. Primi (Ed.), Temas em avaliação psicológica (pp. 7-16). Campinas, SP: Impressão Digital do Brasil.

Primi, R., \& Almeida, L. S. (2000). Baterias de provas de raciocínio (BPR-5): Manual Técnico. São Paulo, SP: Casa do Psicólogo.

Roberts, R. D., Zeidner, M., \& Matthews, G. (2001). Does emotional intelligence meet traditional standards for an 'intelligence'? Some new data and conclusions. Emotion, 1, 196-231.

Salovey, P., \& Mayer, J. D. (1990). Emotional intelligence. Imagination, Cognition and Personality, 9(3), 185-2 11.

Schaie, K. W. (2001). Emotional intelligence: Psycometric status and developmental characteristics - Comment on Roberts, Zeidner, and Matthews. Emotion, 1(3), 243-248.

Sternberg, R. J., Forsythe, G. B., Hedlund, J., Horvath, J. A., Wagner, R. K., Williams, W. M., Snook, S. A., \& Grigorenko, E. L. (2000). Practical intelligence in everyday life. New York: Cambridge University Press.

Wechsler, S. M. (1999). Guia de procedimentos éticos para a avaliação psicológica. In S. M. Wechsler \& R. S. L. Guzzo (Ed.), Avaliação psicológica: Perspectiva internacional (pp. 133141). São Paulo, SP: Casa do Psicólogo. 